\title{
The Nervous System: An Ideal Therapeutic Target for Anti-Schistosomal Drug Discovery
}

\section{Shi-Bin Cheng*}

Department of Pediatrics, Women and Infants Hospital, Alpert Medical School, Brown University, USA

Schistosomiasis is a parasitic disease that afflicts over 200 million people worldwide, causing visceral organ disturbance, and impairing growth and cognitive development in children [1]. The etiological agents of human schistosomiasis are the blood flukes of the genus Schistosoma, including S. mansoni, S. haematobium, and S. japonicum. S. mansoni is blamed for over $90 \%$ of all human schistosomiasis [2]. Epidemiological evidence reveals that schistosomiasis is typically endemic in tropical countries in Africa, the Caribbean, South America, Southeastern Asia and Middle East, especially in the regions where the intermediate snail host that carries the parasite. Because no vaccine is available for Schistosoma, chemotherapeutic intervention is still the primary option for schistosomiasis treatment. The treatment of human schistosomiasis relies solely on Praziquantel which has been widely used for over three decades. However, emergence of drug resistance in the worms has ignited enthusiasm to search for alternative drug candidates.

To date, there have been numerous candidate molecules that were proposed as potential chemotherapeutic targets for treating schistosomiasis. These molecules are involved in a variety of survivalrelated machineries of the worm, including redox metabolism (e.g. thioredoxin glutathione reductase) [3,4], ion channels (e.g. calcium channel subunits) [5], chromatin modification (e.g. histone acetyltransferases and deacetylase) [6], metal homeostasis (e.g. phytochelatin synthase) $[7,8]$, protein maturation (e.g. methionine aminopeptidase) [9], and cell signaling (e.g. cAMP-dependent protein kinase and cyclophilin) [10,11]. In contrast to these potential therapeutic strategies, the nervous system of the helminth parasites has been successfully employed as a target by anthelmintics currently in use, including ivermectin, levamisole and monepantel [1]. All of these drugs act on neuroreceptors in the neuromuscular system of the worm, which results in disrupting the neural and neuromuscular transmission, and consequently paralyzing and killing the worm. Ivermectin not only eliminates nematodes, but also trematodes (Fasciola spp., Schistosoma spp.) as well [1].

Schistosoma has a well developed nervous system that consists of a simple brain and several pairs of longitudinal nerve cords (the central nervous system) and a peripheral network that innervates almost all body tissues, especially the tegument, the somatic musculature and the suckers $[1,2]$. Biogenic amines, including serotonin, dopamine and 5-hydroxytryptamine, are the major neurotransmitters in the nervous system of schistosomes and function as pivotal modulators of neuromuscular transmission, regulating locomotion, attachment to the host and many other behaviors that are essential to the parasite survival in the host [1]. Thus, Biosynthetic enzymes, receptors and transporters of biogenic amines may be aimed as the targets for the development of chemotherapeutic agents against schistosomes. Drug screens have revealed that blockers for serotonergic and dopaminergic receptors and serotonin transporter are the potent disruptors that inhibit schistosome motility [1]. Serotonergic and dopaminergic receptors belong to the members of the super family of G ProteinCoupled Receptors (GPCRs). Recently, Ribeiro et al. have cloned a novel Schistosoma mansoni G protein-coupled receptor (SmGPR-3)
[2]. SmGPR-3 was activated by dopamine and other catecholamines, resulting in a strong effect on the motility of larval schistosomes [2]. Immuno staining showed the localization of SmGPR-3 in the central nervous system and the peripheral plexuses innervating the musculature, the caecum, the tubercles and the male reproductive system of Schistosoma mansoni [2]. Since SmGPR-3 is substantially different from dopamine receptor of the mammalian host, this novel protein may be employed as a potential target for developing new schistosome-specific drugs. However, which G protein is recruited by SmGPR-3, how SmGPR-3 undergoes trafficking, endocytosis and degradation, and how SmGPR-3 signals remain yet to be investigated.

GPCRs constitute the largest family of cell surface receptors which share a common topology of seven transmembrane domains and modulate a variety of cell activities [12]. GPCRs are the targets of nearly half drugs currently in use and for the development of new therapeutics that treat a wide range of human diseases [12]. Regulation of surface expression, endocytosis, recycling and degradation of GPCRs involves a number of machineries that spatiotemporally mediate GPCRspromoted cell signaling $[13,14]$. Thus, all these machineries could be targeted for drug development. Moreover, the significant evolutionary difference in the structures of GPCRs between schistosome and the host allows developing drugs that specifically kill schistosome but cause less side-effect in the host [1]. To date, most of anthelmintic drugs currently in use act on the nervous system of helminth parasites [1]. Likewise, GPCRs in the nervous system of schistosome is a promising therapeutic target for anti-schistosomal drug discovery.

\section{References}

1. Ribeiro P, Gupta V, El-Sakkary N (2012) Biogenic amines and the control of neuromuscular signaling in schistosomes. Invert Neurosci 12: 13-28.

2. El-Shehabi F, Taman A, Moali LS, El-Sakkary N, Ribeiro P (2012) A novel G protein-coupled receptor of Schistosoma mansoni (SmGPR-3) is activated by dopamine and is widely expressed in the nervous system. PLoS Negl Trop Dis 6: e1523.

3. Ross F, Hernández P, Porcal W, López GV, Cerecetto H, et al. (2012) Identification of thioredoxin glutathione reductase inhibitors that kill cestode and trematode parasites. PLoS One 7: e35033.

4. Song L, Li J, Xie S, Qian C, Wang J, et al. (2012) Thioredoxin glutathione reductase as a novel drug target: evidence from Schistosoma japonicum. PLoS One 7: e31456.

*Corresponding author: Shi-Bin Cheng, Department of Pediatrics, Women and Infants Hospital, Alpert, Medical School, Brown University, Providence, RI 02903 USA, Tel: 401-274-1122; E-mail: shibin_cheng@brown.edu

Received January 02, 2013; Accepted January 05, 2013; Published January 28 2013

Citation: Cheng SB (2013) The Nervous System: An Ideal Therapeutic Target for Anti-Schistosomal Drug Discovery. Trop Med Surg 1: e103. doi:10.4172/2329 9088.1000e103

Copyright: (c) 2013 Cheng SB. This is an open-access article distributed under the terms of the Creative Commons Attribution License, which permits unrestricted use, distribution, and reproduction in any medium, provided the original author and source are credited. 
Citation: Cheng SB (2013) The Nervous System: An Ideal Therapeutic Target for Anti-Schistosomal Drug Discovery. Trop Med Surg 1: e103. doi:10.4172/2329-9088.1000e103

Page 2 of 2

5. Salvador-Recatalà V, Greenberg RM (2012) Calcium channels of schistosomes: unresolved questions and unexpected answers. Wiley Interdiscip Rev Membr Transp Signal 1: 85-93.

6. Pierce RJ, Dubois-Abdesselem F, Caby S, Trolet J, Lancelot J, et al. (2011) Chromatin regulation in schistosomes and histone modifying enzymes as drug targets. Mem Inst Oswaldo Cruz 106: 794-801.

7. Ray D, Williams DL (2011) Characterization of the phytochelatin synthase of Schistosoma mansoni. PLoS Negl Trop Dis 5: e1168.

8. Glanfield A, McManus DP, Anderson GJ, Jones MK (2007) Pumping iron: a potential target for novel therapeutics against schistosomes. Trends Parasito 23: $583-588$

9. Peng J, Han H, Hong Y, Fu Z, Liu J, et al. (2010) Molecular cloning and characterization of a gene encoding methionine aminopeptidase 2 of Schistosoma japonicum. Parasitol Res 107: 939-946.
10. Han H, Peng J, Hong Y, Fu Z, Xu J, et al. (2012) Molecular cloning and characterization of a cyclophilin A homologue from Schistosoma japonicum. Parasitol Res 111: 807-817.

11. Swierczewski BE, Davies SJ (2009) A schistosome cAMP-dependent protein kinase catalytic subunit is essential for parasite viability. PLoS Negl Trop Dis 3: e505.

12. Marchese A, Paing MM, Temple BR, Trejo J (2008) G protein-coupled receptor sorting to endosomes and lysosomes. Annu Rev Pharmacol Toxicol 48: 601 629 .

13. Cheng SB, Filardo EJ (2012) Trans-Golgi Network (TGN) as a regulatory node for $\beta 1$-adrenergic receptor ( $\beta 1 A R$ ) down-modulation and recycling. J Biol Chem 287: 14178-1491.

14. Cheng SB, Quinn JA, Graeber CT, Filardo EJ (2011) Down-modulation of the G-protein-coupled estrogen receptor, GPER, from the cell surface occurs via a trans-Golgi-proteasome pathway. J Biol Chem 286: 22441-22455. 